\title{
Identification of active galaxies behind the Coma cluster of galaxies $^{\star}$
}

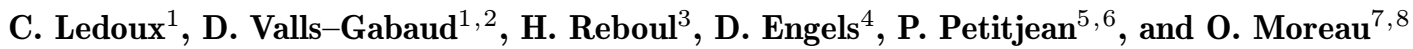 \\ 1 UMR 7550 CNRS, Observatoire Astronomique de Strasbourg, 11 rue de l'Université, F-67000 Strasbourg, France \\ 2 Institute of Astronomy, Madingley Road, Cambridge CB3 0HA, UK \\ 3 GRAAL, Université Montpellier II, F-34095 Montpellier Cedex 5, France \\ 4 Hamburger Sternwarte, Gojenbergsweg 112, D-21029 Hamburg, Germany \\ 5 Institut d'Astrophysique de Paris - CNRS, 98bis Boulevard Arago, F-75014 Paris, France \\ 6 DAEC, Observatoire de Paris-Meudon, F-92195 Meudon Principal Cedex, France \\ 7 Laboratoire d'Astronomie de l'Université Lille I, Impasse de l'Observatoire, F-59000 Lille, France \\ 8 Centre d'Analyse des Images, Observatoire de Paris, 61 avenue de l'Observatoire, F-75014 Paris, France
}

Received January 12; accepted May 21, 1999

\begin{abstract}
We describe the results of an efficient survey to identify bright $B \lesssim 18$ active galactic nuclei (AGN) within $\approx 4^{\circ}\left(4.7 h^{-1} \mathrm{Mpc}\right.$ projected radius $)$ of the centre of the Coma cluster of galaxies. The candidates have been carefully selected either as unresolved UV-excess objects with no detected proper motion, or from identifications on objective-prism plates including a few ROSAT sources. A global success rate larger than $30 \%$ is achieved. Low-resolution spectra are presented for the extragalactic objects identified in the course of the survey: the sample contains 16 emission-line objects, a BL Lac candidate and an elliptical galaxy. The AGNs of this list are unique targets to study the association of the Lyman- $\alpha$ forest with large-scale structures and galactic haloes at low redshift, to search for warm gas within the Coma cluster and nearby filaments, and for optical monitoring to detect the microlensing variability produced by the baryonic dark matter in the cluster.
\end{abstract}

Key words: quasars: general - quasars: absorption lines - galaxies: Seyfert - galaxies: starburst techniques: spectroscopic — surveys

\section{Introduction}

Bright quasars (QSOs) can be used as background sources to probe the physical properties of foreground objects.

Send offprint requests to: C. Ledoux

(cedric@astro.u-strasbg.fr)

* Based on observations carried out at the $1.93 \mathrm{~m}$ telescope of the Observatoire de Haute-Provence (CNRS), France.
When the foreground is a cluster of galaxies, the quasars behind the cluster can be used to map not only the warm gas content of the intra-cluster medium (via absorption lines), but also its dark matter distribution (via microlensing).

The discovery of a diffuse extreme UV-excess in nearby clusters by the Extreme Ultraviolet Explorer (EUVE) satellite (Lieu et al. 1996a,b) was initially interpreted as evidence for a warm gas component at about $500000 \mathrm{~K}$ in the intra-cluster medium. This would constitute a major mass component and raises enormous problems (Fabian 1996). A non-thermal explanation, based on inverse Compton scattering, seems more plausible and consistent with the hard X-ray tail observed by BeppoSAX (Ensslin \& Biermann 1998; Sarazin \& Lieu 1998). However the distorted shape of the EUV isophotes may be more consistent with a shock heated medium, so the situation remains very confused. Although attempts have been made to detect the warm gas through UV emission lines (Dixon et al. 1996), absorption lines are far more sensitive to the presence of this component. Hence, Hubble Space Telescope (HST) spectroscopy of background QSOs should reveal absorption lines at the redshift of the cluster if the thermal interpretation is correct.

In addition, background QSOs can be used as probes of intervening gaseous clouds and of their evolution along the line of sight. Adjacent lines of sight provided by close pairs of QSOs can also constrain the size of the absorbers, their spatial distribution and their connection with galaxies. In this context, targeting quasars behind low-redshift clusters is a very efficient way to reveal the connection of the intergalactic medium on any scale with these 
concentrations of galaxies. Coma (Abell 1656, at a redshift of $\left\langle z_{\text {Coma }}\right\rangle=0.0229$ ) is ideal in this respect since it is one of the best studied areas of the sky as far as redshift coverage and galaxy properties are concerned (Biviano 1998).

Quasar pairs with projected separations of no more than a few arcminutes yield interesting constraints on the size, physical structure and kinematics of galactic haloes, clusters and filaments (Smette et al. 1995; Petitjean et al. 1998; D'Odorico et al. 1998). The ideal project however would be to observe a large number of QSOs in a small solid angle on the sky to probe all scales at the same time. Since absorption is extremely sensitive to the total amount of warm gas, this would probe even small column densities inside the cluster and also reveal, in the outskirts of the cluster, the connection with the large-scale filamentary network (Cen et al. 1994; Petitjean et al. 1995; Hernquist et al. 1996; Miralda-Escudé et al. 1996).

Besides the mapping of the warm gas in the cluster and in the neighbouring environment, a third goal of a QSO survey behind clusters is the mapping of their baryonic dark matter content. This can be achieved by monitoring the background QSOs searching for the expected micro-lensing signature in their light curves. The micro-lensing optical depth produced by a cluster is very large, a few $10^{-3}$, and for lens masses ranging from $10^{-5}$ to $10^{-3} M_{\odot}$ the events will have typical time scales of days (Walker \& Ireland 1995; Tadros et al. 1998; Wu \& Xue 1998). A program to monitor a few tens of QSOs behind Coma for instance, for 5 months with a daily sampling rate, should reveal dozens of micro-lensing events and produce a 2-D mapping of the baryonic dark matter within the cluster.

There are however very few quasars known to date behind low-redshift clusters and clearly additional background quasars, and more generally galaxies with an active galactic nucleus, are badly needed for all these studies. These sources have to be bright enough to be accessible for medium-resolution spectroscopy with the HST and for optical monitoring with small/medium-size ground-based telescopes.

Until now optical searches for quasars have only been performed systematically behind Virgo (He et al. 1984; Impey \& He 1986), where 29 QSOs were found out of 82 candidates observed at the Palomar 5-meter telescope. There are a few X-ray selected QSOs found in ROSAT images whose optical counterparts were identified in the Digitized Sky Survey around several clusters (Knezek \& Bregman 1998). Although interesting, this technique only provides a handful of QSOs in the vicinity of clusters. There are also unpublished surveys around Abell 2029 and Abell 2255 (Horowitz et al. 1994). We report here on a survey (Ledoux et al. 1998) to complete the extant catalogues of QSOs/AGNs within a projected radius of $\approx 4^{\circ}$ from the centre of the Coma cluster, down to a limiting magnitude of about $B<18$. We describe the selection methods in Sect. 2 and present the observations in Sect. 3. In Sects. 4 and 5, we discuss the characteristics of the extragalactic objects identified in this way, and conclude in Sect. 6 on the global efficiency of the selection.

Throughout this paper, we assume a standard Einstein-De Sitter cosmological model and a Hubble constant $H_{0}=100 h \mathrm{~km} \mathrm{~s}^{-1} \mathrm{Mpc}^{-1}$.

\section{Target selection}

AGN candidates were selected with the criterion $B \lesssim 18$ from 3 different sources: [1] the OMHR survey of UV-excess objects at the North Galactic pole (Moreau \& Reboul 1995), [2] the Hamburg Quasar Survey (HQS) by Hagen et al. (1995) and [3] the ROSAT All-Sky Survey (RASS) using identifications on HQS plates (Bade et al. 1998).

We also selected the object US 370 (Usher 1981), which was classified as a QSO candidate with $V=18$ and $U-V=-0.55$ by Berger et al. (1991). The proper motion of this target could not be measured due to the proximity of a bright star as mentioned by Moreau \& Reboul (1995). A slit-less observation was performed by Weedman (1985) and we provide here a spectrum of US 370 to confirm its nature and measure its redshift properly.

\subsection{OMHR candidates}

Berger et al. (1991) performed a photometric $(U, B, V)$ and astrometric analysis of $1221 \mathrm{UV}$-excess unresolved objects in a $40.5^{\square}$ field almost centred on the selected area SA 57:

$$
\begin{aligned}
12^{\mathrm{h}} 52^{\mathrm{m}} 08.6^{\mathrm{s}} & <\alpha_{\mathrm{J} 2000}<13^{\mathrm{h}} 21^{\mathrm{m}} 39.2^{\mathrm{s}} \\
26^{\circ} 01^{\prime} 12^{\prime \prime} & <\delta_{\mathrm{J} 2000}<32^{\circ} 14^{\prime} 39^{\prime \prime} .
\end{aligned}
$$

They used the MAMA (Machine Automatique à Mesurer pour l'Astronomie) machine to scan three plates taken in 1962 at the Palomar 48-inch Schmidt. This field was fully reduced in $U, B$ and $V$ by Moreau \& Reboul (1995) with a visual limiting magnitude 20.3. Amongst the $\sim 130000$ detected objects, the authors extracted a list of 1759 quasar candidates selected mainly as UV-excess unresolved objects. The colour criterion was $U-V<0.1$ with a typical accuracy of 0.1 for the $U$ and $V$ magnitudes. After rejection of the objects with previous spectroscopic identifications, a total of 1681 quasar candidates remained.

A recent comparison of the MAMA catalogues extracted from the Schmidt plates taken in 1962 with those obtained in the same way from OCA (Observatoire de la Côte d'Azur) Schmidt plates taken in 1991 and 1994 provides measures of proper motions of the above OMHR quasar candidates, among many other objects (Moreau \& Reboul, in preparation). The limited field of view of the 
OCA telescope constrained this proper motion survey to a $26.7^{\square}$ field within:

$$
\begin{aligned}
12^{\mathrm{h}} 56^{\mathrm{m}} 42.1^{\mathrm{s}} & <\alpha_{\mathrm{J} 2000}<13^{\mathrm{h}} 20^{\mathrm{m}} 41.4^{\mathrm{s}} \\
26^{\circ} 50^{\prime} 42^{\prime \prime} & <\delta_{\mathrm{J} 2000}<31^{\circ} 57^{\prime} 51^{\prime \prime} .
\end{aligned}
$$

The $1 \sigma$ sensitivity of the measurement of proper motions

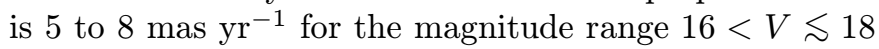
used in the present work.

The combined requirements of [1] unresolved morphology, [2] UV-excess and [3] absence of proper motion larger

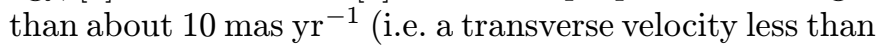
$25 \mathrm{~km} \mathrm{~s}^{-1}$ at $500 \mathrm{pc}$ ) give a priori high-quality quasar candidates. However, the constraint imposed by medium resolution spectroscopy with the HST restricted our choice to objects brighter than magnitude 18. Quasars this bright are drastically scarcer (and consequently samples are far more polluted by stars) than for visual magnitudes fainter than $V=18$ where the best and most numerous quasar candidates are expected to lie.

The sample of OMHR objects selected for observation finally contained the 18 brightest and bluest targets $(B \lesssim 18, U-V \leq 0$ ) with no proper motion detected at the 0.68 confidence level.

\section{2. $H Q S$ and $R A S S$ candidates}

Quasar candidates were selected from the HQS digitized objective-prism plates in two steps (Hagen et al. 1995, 1999). In the first step, prism spectra with a blue continuum are selected automatically from the digitized data which are available only at low resolution. The selected spectra are rescanned with the Hamburg PDS machine to yield high-resolution spectra having a dispersion of $139 \mathrm{~nm} \mathrm{~mm}^{-1}$ at $\mathrm{H} \gamma$. These spectra are interactively classified on a vector graphics screen. Non-blue spectra with stellar absorption features are discarded while the others are kept in an archive.

Blue stellar-like spectra are classified as "QSO/HS", meaning quasar or hot star candidate. Spectra with apparent emission lines or unusual continua are classified as "QSO" or "Narrow Emission". If the spectra are blue and featureless they enter the category "Unid" for "unidentified". The number of entries in these classes increases towards the "unidentified" sources, while the efficiency to find quasars in these classes decreases from "QSO" towards the other classes.

We selected from the archive all the objects with $B \leq 18$ contained in the above mentioned classes within about $4^{\circ}$ from the centre of the Coma cluster. Hagen et al. (1999) estimate that for $z>0.1$ and $B \leq 17.5$ less than $10 \%$ of the quasars are missed. Objects already known from the literature were discarded by cross-correlation with the NED and SIMBAD databases. We checked for overlaps with the OMHR survey, and discarded as well the objects with a significant proper motion (confidence level $>0.68$ ) or colour $U-V>0$. The final HQS list used to obtain follow-up spectroscopy contains 31 objects.

The HQS list was augmented by two AGN candidates from the RASS. X-ray sources from this survey are identified on the Schmidt plates of the HQS. The confirmation rate of the AGN candidates is very high, about $>90 \%$ (Bade et al. 1992), but most of them are optically fainter than our selection limit or were already known. The first RASS source RXJ1252.6+3002 will be contained in the RASS Faint Source Catalog (Voges et al., in preparation) while the second one RXJ1303.7+2633 is published in the RASS Bright Source Catalogue (Voges et al. 1997). Also, HS $1312+2735$ is listed as X-ray source in the latter catalogue (namely RXJ1314.3+2719).

\section{Optical spectroscopy}

The spectroscopic observations aimed at confirming the nature of the selected candidates were carried out at the F/15 Cassegrain focus of the $1.93 \mathrm{~m}$ telescope at the Observatoire de Haute-Provence (CNRS, France) during the nights of April 8-15, 1997 and April 20-21, 27-29, 1998. The long-slit spectrograph CARELEC (Lemaitre et al. 1990) was operated with a 150 lines $\mathrm{mm}^{-1}$ grating leading to a dispersion of $26 \mathrm{~nm} \mathrm{~mm}^{-1}$ in the wavelength range $\lambda \lambda 380.0-725.0 \mathrm{~nm}$. The detector was a $512 \times 512$ Tektronix CCD with $27 \mu \mathrm{m}$ pixels and a rms read-out noise of 9.2 electrons. Throughout the observing runs the atmospheric seeing usually ranged from $1^{\prime \prime} .5$ to 2 .' 0 . Most of the observations were made with a 2 .'5 slit width, which corresponds to a projected slit width on the detector of $62 \mu \mathrm{m}$ or 2.3 pixels. With this set-up, the overall instrumental resolution FWHM was $1.4 \mathrm{~nm}$ as measured on the He arc lines used for the wavelength calibration. An exposure time of $3600 \mathrm{~s}$ was enough to yield a signal-to-noise ratio larger than 10 in the spectra of the faintest candidates $(B \approx 18)$.

The sub-dwarf O star HZ 44 (Oke 1990) was used as a spectro-photometric standard during both observing runs. Depending on the atmospheric stability of the night, the absolute flux calibration is uncertain at the 20 to $50 \%$ level. The data were reduced and analyzed independently by two of us, with the LONG-SLIT package implemented within MIDAS (CL), and with IRAF (DVG). After flat-field correction and wavelength calibration, the object signal was extracted using the Horne algorithm (Horne 1986). It ensures an optimal signal-to-noise ratio for weak spectra while it possibly slightly underestimates the emission-line strength in extended sources.

\section{Sample characteristics}

The coordinates, apparent magnitudes and redshifts of the extragalactic objects are listed in Table 1. 

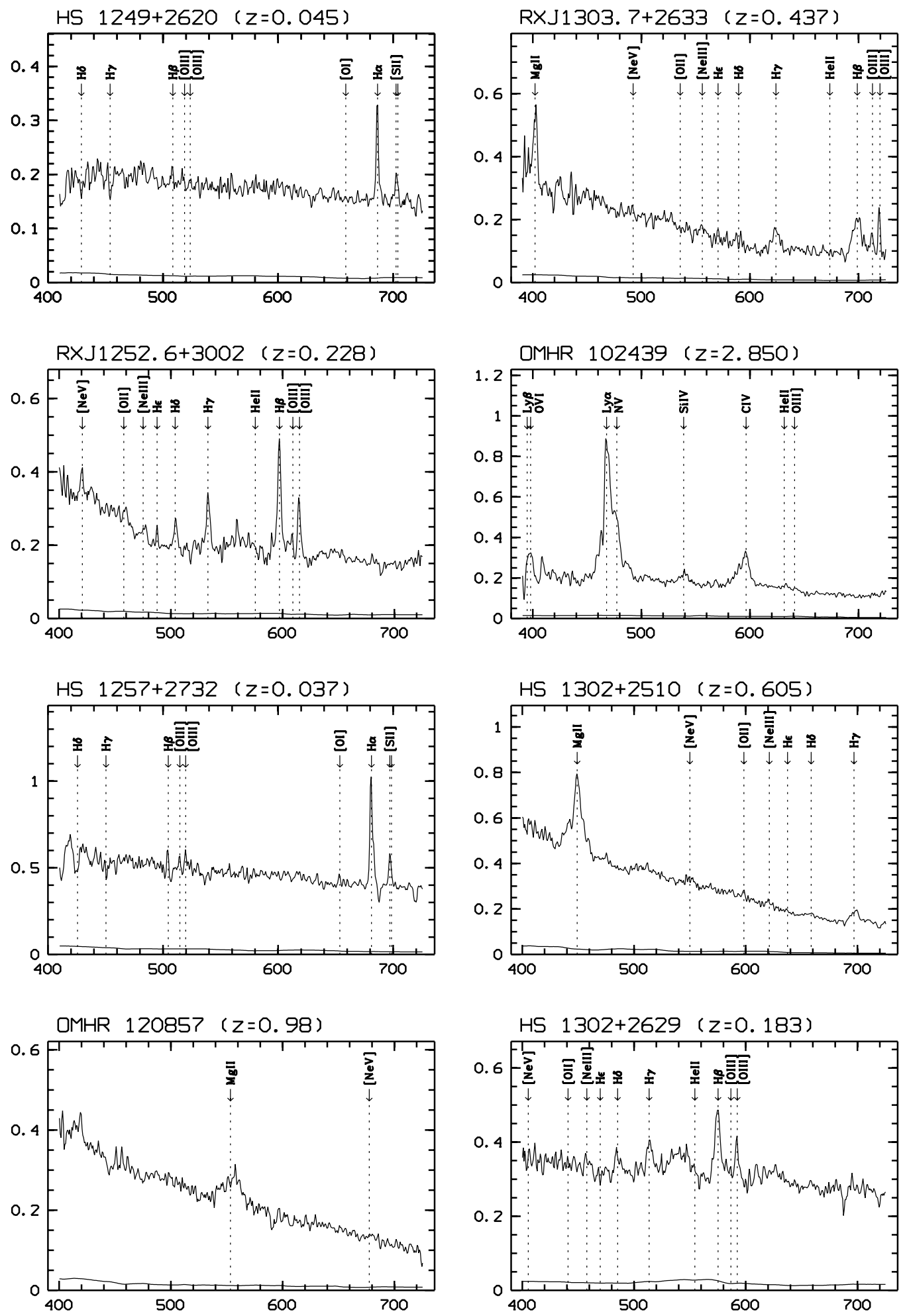

Fig. 1. Optical spectra of the extragalactic objects at a resolution FWHM of $1.4 \mathrm{~nm}$ : the calibrated flux $F_{\lambda}$ in units of $10^{-17} \mathrm{~W}$ $\mathrm{m}^{-2} \mathrm{~nm}^{-1}$ is plotted against heliocentric wavelength $\lambda$ in $\mathrm{nm}$. The curve below each spectrum is the associated $1 \sigma$ standard deviation of the noise in the same flux units 

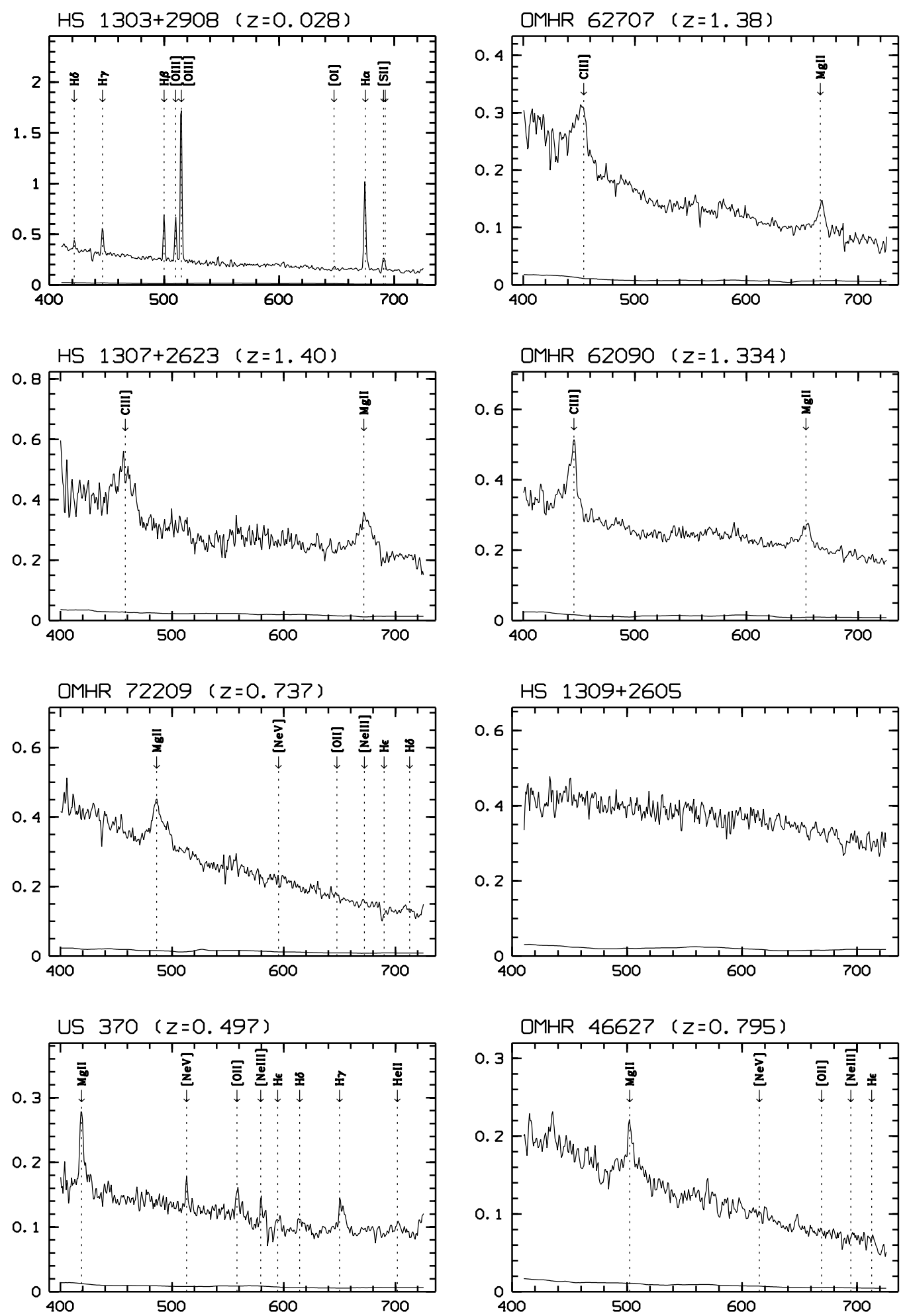

Fig. 1. continued 

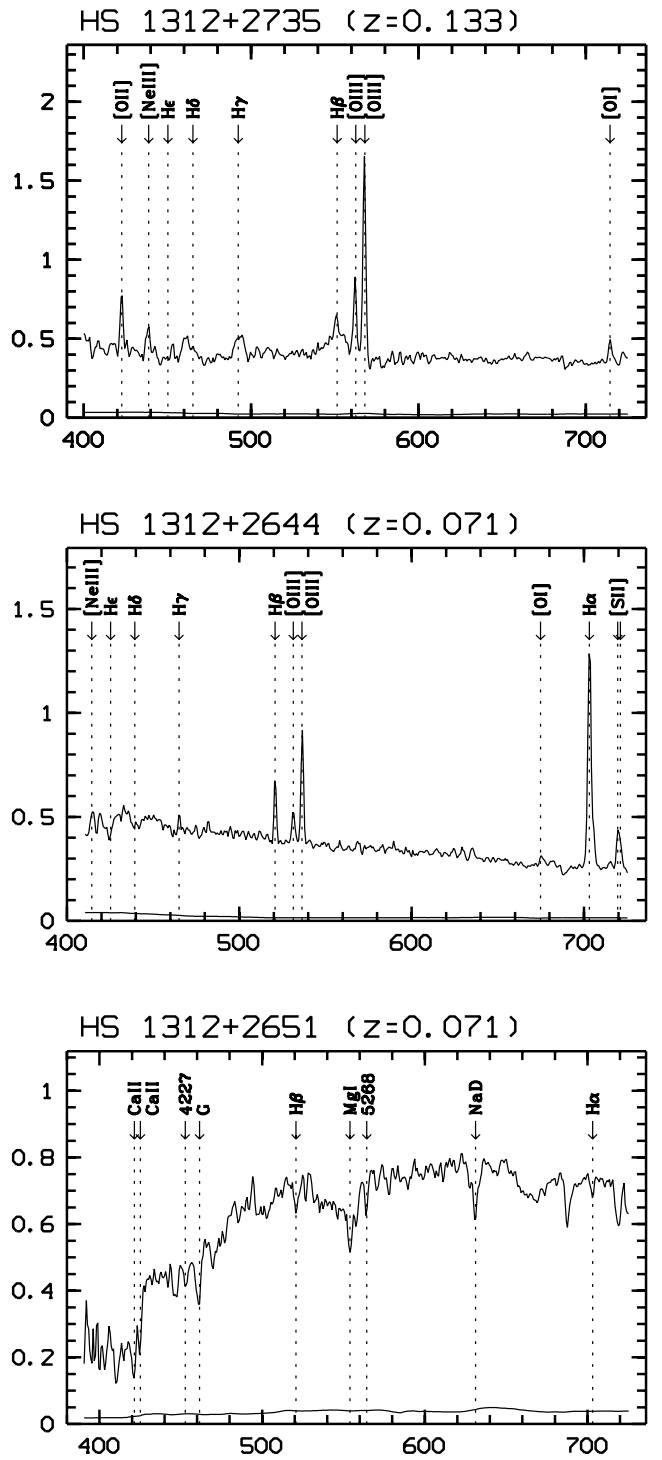

Fig. 1. continued

The projected impact parameters (Col. 6) range from 0.8 to about $4.7 h^{-1} \mathrm{Mpc}$, and therefore probe the cluster from the inner Abell radius all the way out to the low-density environment and large-scale filaments. The wavelength and flux calibrated 1-D spectra are displayed in Fig. 1. The mean signal-to-noise ratio has been computed as a function of wavelength from the photon statistics after subtraction of the spectral lines in the normalized spectrum. Besides the stellar objects (which are dealt with in a separate paper), we discovered 16 new emission-line objects, a BL Lac candidate and an elliptical galaxy. We confirmed the redshift of the QSO US 370. The emission-line objects consist of 9 quasars, 3 Seyfert 1 and 4 Hil-region galaxies (see below). We included HS $1309+2605$ as a BL Lac candidate because none of the usual stellar absorption features could be detected in the slit spectrum (see Fig. 1). However there has been no radio detection (Kim et al. 1994; Kim 1994) and the signal-to-noise ratio in our optical spectrum is insufficient for a final decision. The normal galaxy HS $1312+2651$ was originally classified as a "Narrow Emission" object according to the objective-prism spectrum, but the emission feature could not be confirmed.

Twelve emission-line objects show broad permitted emission lines and should be quasars or Seyfert 1 galaxies. We adopt the definition by Véron-Cetty \& Véron (1998) of a quasar as a star-like object, or an object with a starlike nucleus, brighter than the absolute $B$-band magnitude of $-21.5+5 \log h$. We have made a distinction between QSOs and Seyfert 1 galaxies on the basis of this definition, and calculated the absolute $B$-band magnitude $M_{B}$ of all the objects in Table 1 (Col. 10). Note that no Kcorrection was applied since it is usually of the same order as the uncertainties in the photometry and/or it does not affect the classification.

Among the Seyfert galaxies, we find several sub-types. RXJ1252.6+3002 belongs to the class of narrow-line Seyfert 1 galaxies (Boller et al. 1996). The intrinsic FWHM of $\mathrm{H} \beta$, around $1150 \mathrm{~km} \mathrm{~s}^{-1}$, is typical for such objects, and it is only slightly larger than that of $[\mathrm{OIII}] \lambda 500.7$. Moreover, the flux ratio of $[\mathrm{OIII}] \lambda 500.7$ to $\mathrm{H} \beta$ is less than unity which rules out the possibility that it is a Seyfert 2 (Shuder \& Osterbrock 1981). Additional support for this classification comes from the detection of strong blends of FeII lines in the regions around $457.0 \mathrm{~nm}$ and $519.0 \mathrm{~nm}$ (Koski 1978). HS 1302+2629 also shows relatively narrow lines, but given its FWHM at $\mathrm{H} \beta$ of $\simeq 2350 \mathrm{~km} \mathrm{~s}^{-1}$, it has to be classified as a classical Seyfert 1 galaxy. Although HS $1312+2735$ is obviously a Seyfert 1 galaxy, a Seyfert 1.5 type (Netzer 1990) is still possible given the shape of the $\mathrm{H} \beta$ line.

The intrinsically faintest objects HS $1249+2620$, HS $1257+2732$, HS $1303+2908$ and HS $1312+2644$ only show narrow permitted emission lines. In Sect. 5 below, we show that these narrow emission-line galaxies (NELGs) are indeed HiI region (i.e. starburst) galaxies.

\section{Redshifts and line parameters}

Due to the large wavelength coverage of the spectra, more than one emission line is detected in most of the cases of objects classified as QSOs, Seyfert 1 galaxies or NELGs, providing secure identifications. When possible three prominent emission lines were selected, preferentially unresolved features far from the border of the spectra where the wavelength calibration is possibly less reliable. A Gaussian profile was fitted to those lines (see Table 1, Col. 8) so as to determine their centroid, and the AGN's redshift was computed by averaging the redshift of the individual lines. In Table 1 (Col. 9), redshift values are vacuum and given in the heliocentric rest frame. The formal error on them is typically of the order of $0.1 \mathrm{~nm}$ or $\Delta z \sim 10^{-3}$, except for OMHR 120857 and HS 1307+2623 where one (or two) broad and somewhat asymmetric line(s) lead(s) to a larger centering error (up to $\Delta z=0.01$ ). 
Table 1. The sample of confirmed extragalactic sources

\begin{tabular}{|c|c|c|c|c|c|c|c|c|c|c|}
\hline $\mathrm{Nr}$ & Object & $\begin{array}{c}\alpha(\mathrm{J} 2000) \\
(\mathrm{h} \mathrm{m} \mathrm{s}) \\
(3)\end{array}$ & 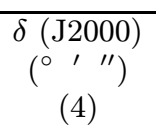 & $\begin{array}{c}\theta_{\text {Coma }} \\
\left(\begin{array}{c}\left.{ }^{\circ}\right) \\
(5)\end{array}\right.\end{array}$ & $\begin{array}{c}\rho_{\text {Coma }}^{\mathrm{a}} \\
\left(h^{-1} \mathrm{Mpc}\right) \\
(6)\end{array}$ & $B^{\mathrm{b}}$ & $\begin{array}{c}\text { Lines } \\
(8)\end{array}$ & $\begin{array}{r}z_{\text {helio }} \\
\quad(9)\end{array}$ & $\begin{array}{c}M_{B}^{\mathrm{c}} \\
-5 \log h \\
(10)\end{array}$ & Type \\
\hline 1 & HS $1249+2620$ & 125154.4 & +260359 & 2.58 & 3.0 & 17.5 & $\mathrm{H} \beta, \mathrm{H} \alpha$ & 0.045 & -18.2 & HII \\
\hline 2 & RXJ1252.6+3002 & 125237.8 & +300213 & 2.58 & 3.0 & 18.2 & $\mathrm{H} \gamma, \mathrm{H} \beta,[\mathrm{OIII}]$ & 0.228 & -21.1 & NLSy1 \\
\hline 3 & HS $1257+2732$ & 130014.4 & +271649 & 0.70 & 0.8 & 16.0 & {$[\mathrm{OIII}], \mathrm{H} \alpha$} & 0.037 & -19.2 & HII \\
\hline 4 & OMHR 120857 & 130203.1 & +265809 & 1.13 & 1.3 & 17.1 & MgII & $0.98:$ & -25.6 & QSO \\
\hline 5 & RXJ1303.7+2633 & 130346.0 & +263311 & 1.68 & 1.9 & 17.8 & $\mathrm{MgII}, \mathrm{H} \gamma, \mathrm{H} \beta$ & 0.437 & -23.0 & QSO \\
\hline 6 & OMHR 102439 & 130412.0 & +295349 & 2.16 & 2.5 & 18.1 & Ly $\alpha$, Sirv, Civ & 2.850 & -27.2 & QSO \\
\hline 7 & HS $1302+2510$ & 130451.3 & +245447 & 3.27 & 3.8 & 17.4 & MgII, [OII] & 0.605 & -24.1 & QSO \\
\hline 8 & HS $1302+2629$ & 130516.7 & +261303 & 2.15 & 2.5 & 17.6 & $\mathrm{H} \gamma, \mathrm{H} \beta,[\mathrm{OIII}]$ & 0.183 & -21.2 & Sy1 \\
\hline 9 & HS $1303+2908$ & 130545.1 & +285212 & 1.60 & 1.8 & 16.8 & $\mathrm{H} \beta,[\mathrm{OIII}]$ & 0.028 & -17.8 & HII \\
\hline 10 & HS $1307+2623$ & 130928.8 & +260748 & 2.85 & 3.3 & 17.7 & CIII],MgII & 1.40 & -25.8 & QSO \\
\hline 11 & OMHR 72209 & 130944.9 & +282735 & 2.26 & 2.6 & 17.9 & MgII & 0.737: & -24.1 & QSO \\
\hline 12 & US $370^{\mathrm{d}}$ & 131028.1 & +280838 & 2.38 & 2.7 & 17.9 & $\mathrm{MgII},[\mathrm{Nev}],[\mathrm{OII}]$ & 0.497 & -23.2 & QSO \\
\hline 13 & OMHR 62707 & 131059.0 & +300107 & 3.20 & 3.7 & 18.1 & CIII],MgII & 1.38 & -25.4 & QSO \\
\hline 14 & OMHR 62090 & 131128.4 & +310551 & 4.04 & 4.7 & 18.0 & CIII],MgII & 1.334 & -25.4 & QSO \\
\hline 15 & HS $1309+2605$ & 131219.2 & +254958 & 3.53 & 4.1 & 17.8 & $\ldots$ & $\ldots$ & $\ldots$ & BL Lac: \\
\hline 16 & OMHR 46627 & 131336.2 & +295815 & 3.64 & 4.2 & 18.1 & MgII & 0.795: & -24.1 & QSO \\
\hline 17 & HS $1312+2735$ & 131423.5 & +271920 & 3.31 & 3.8 & 17.2 & [OIII] & 0.133 & -20.9 & Sy1 \\
\hline 18 & HS $1312+2644$ & 131431.9 & +262831 & 3.62 & 4.2 & 17.0 & $\mathrm{H} \beta,[\mathrm{OIII}]$ & 0.071 & -19.7 & HII \\
\hline 19 & HS $1312+2651$ & 131502.1 & +263543 & 3.67 & 4.2 & 17.4 & $\mathrm{H} \beta, \mathrm{MgI}, \mathrm{H} \alpha$ & 0.071 & -19.3 & $\mathrm{E}$ \\
\hline
\end{tabular}

a Adopting $<z_{\text {Coma }}>=6853 \mathrm{~km} \mathrm{~s}^{-1}$.

b The magnitudes of the HQS and RASS sources were determined on the objective-prism plates and may have errors up to $0.5 \mathrm{mag}$; the OMHR sources have typical errors in $B$ of $0.1 \mathrm{mag}$.

c No K-correction is applied.

${ }^{\mathrm{d}}$ Usher (1981).

Table 2 displays the measurements of the line parameters for the entire sample of emission-line objects. The numbers of Col. 1 are the same as in Col. 1 of Table 1. The equivalent width $W_{\lambda_{\mathrm{r}}}$ of the detected lines (Col. 3) and their full width at half-maximum FWHM (Col. 4) are both corrected from redshift (rest frame values); FWHM is in addition corrected for the instrumental response. No FWHM value is given for unresolved lines.

The classification of the NELGs can be made on the basis of diagnostic diagrams which discriminate between different physical processes (Baldwin et al. 1981): either photoionization by a non-thermal power-law continuum in a narrow-line AGN (i.e. Seyfert 2 galaxies and LINERS), or photoionization due to UV photons emitted by hot OB stars (i.e. HII region galaxies). We have computed flux ratios for the most important diagnostic lines (Veilleux \& Osterbrock 1987). Criteria involving ratios between adjacent lines were used to get rid as much as possible of extinction problems. From the $[\mathrm{OIII}] / \mathrm{H} \beta-[\mathrm{SII}] / \mathrm{H} \alpha$ diagram, all 4 NELGs turn out to be unambiguously objects with a Hir-like spectrum. The diagnostics based on $[\mathrm{OIII}] / \mathrm{H} \beta-[\mathrm{NII}] / \mathrm{H} \alpha$ and $[\mathrm{OIII}] / \mathrm{H} \beta-[\mathrm{OI}] / \mathrm{H} \alpha$ diagrams are a bit less certain given the errors in the measured fluxes, but give results which are consistent with this classification.

\section{Discussion}

The selection of HQS candidates led to an efficiency of $29 \% \pm 10 \%$ taking into account the probable BL Lac object HS $1309+2605$. Similarly, the $U-V \leq 0$ OMHR candidates with undetected proper motion gave a success rate for identified QSOs of $33 \% \pm 14 \%$.

The OMHR selection actually gives a success rate of $54 \% \pm 14 \%$ if the 8 previously known QSOs falling under the same selection criteria are included, which is a good score for this range of (bright) optical magnitudes. Similarly, deep pointed ROSAT surveys towards low galactic neutral hydrogen column density regions (Shanks et al. 1991; Hasinger et al. 1993, 1994) give a selection of AGNs in X-rays that is very successful with typical efficiencies of up to $\sim 60 \%$ (Zickgraf et al. 1997). We also note that the fact that all OMHR targets are either stars or bona fide QSOs confirms the quality of the diffuse-object rejection by Moreau \& Reboul (1995).

Out of a total of 51 selected candidates, 16 new active galaxies (AGNs and Hir region galaxies) have been identified, and a probable BL Lac object discovered. This leads to a $33 \% \pm 8 \%$ global selection efficiency. Compared to the catalogue of Véron-Cetty \& Véron (1998), the number of confirmed QSOs/AGNs brighter than $18^{\text {th }}$ magnitude $^{1}$ has increased by $22 \%$ within the inner $2^{\circ}$,

\footnotetext{
1 Note however that some sources are ambiguous, like BG 57 27, BG 5749 and BG 5742.
} 
Table 2. Emission-line measurements

\begin{tabular}{|c|c|c|c|c|c|c|c|c|c|c|c|c|c|}
\hline $\mathrm{Nr}$ & $\begin{array}{c}\lambda_{\text {helio }} \\
(\mathrm{nm}) \\
(2)\end{array}$ & $\begin{array}{c}W_{\lambda_{\mathrm{r}}} \\
(\mathrm{nm}) \\
(3)\end{array}$ & $\begin{array}{c}F W H M \\
\left(\mathrm{~km} \mathrm{~s}^{-1}\right) \\
(4)\end{array}$ & $S / N$ & \multicolumn{2}{|c|}{$\begin{array}{c}\text { Transition } \\
(6)\end{array}$} & $\mathrm{Nr}$ & $\begin{array}{c}\lambda_{\text {helio }} \\
(\mathrm{nm}) \\
(2)\end{array}$ & $\begin{array}{c}W_{\lambda_{\mathrm{r}}} \\
(\mathrm{nm}) \\
(3)\end{array}$ & $\begin{array}{c}F W H M \\
\left(\mathrm{~km} \mathrm{~s}^{-1}\right) \\
(4)\end{array}$ & $\overline{S / N}$ & \multicolumn{2}{|c|}{$\begin{array}{c}\text { Transition } \\
(6)\end{array}$} \\
\hline 1 & 508.0 & 0.4 & $\ldots$. & 16 & $\mathrm{H} \beta$ & & 9 & 499.8 & 3.0 & . & 21 & $\mathrm{H} \beta$ & \\
\hline 1 & 523.5 & 0.2 & 987 & 16 & [OıII] & 500.7 & 9 & 509.9 & 3.4 & $\ldots .$. & 20 & [ÖıI $]$ & 495.9 \\
\hline 1 & 686.2 & 3.1 & 550 & 20 & $\mathrm{H} \alpha$ & & 9 & 514.7 & 11.7 & $\ldots .$. & 18 & [Öні $]$ & 500.7 \\
\hline 1 & 702.9 & 1.1 & 1023 & 20 & [SII] & 672.5 & 9 & 674.5 & 11.1 & $\ldots .$. & 25 & $\mathrm{H} \alpha$ & \\
\hline 2 & 420.3 & 0.4 & 1308 & 14 & {$[\mathrm{NeV}]$} & 342.6 & 9 & 691.0 & 1.8 & 733 & 24 & [SII] & 672.5 \\
\hline 2 & 504.2 & 1.0 & 1264 & 16 & $\mathrm{H} \delta$ & & 10 & 456.2 & 3.2 & $7306^{*}$ & 15 & Cini] & 1909 \\
\hline 2 & 533.4 & 2.4 & 1549 & 15 & $\mathrm{H} \gamma$ & & 10 & 673.0 & 2.1 & 2101 & 18 & MgII & 279.8 \\
\hline 2 & 597.0 & 6.8 & 1146 & 15 & $\mathrm{H} \beta$ & & 11 & 486.1 & 2.9 & 5948 & 34 & MgII & 279.8 \\
\hline 2 & 614.7 & 2.7 & 1090 & 20 & [ÖII] & 500.7 & 12 & 418.9 & 3.5 & 1628 & 13 & MgII & 279.8 \\
\hline 3 & 504.0 & 0.6 & 746 & 13 & $\mathrm{H} \beta$ & & 12 & 513.0 & 0.6 & $\ldots .$. & 20 & {$[\mathrm{NeV}]$} & 342.6 \\
\hline 3 & 519.5 & 0.4 & 842 & 13 & [OІІІ] & 500.7 & 12 & 558.5 & 0.8 & 858 & 14 & [OII] & 372.7 \\
\hline 3 & 680.9 & 4.1 & 1040 & 31 & $\mathrm{H} \alpha$ & & 12 & 579.8 & 0.5 & $\ldots .$. & 13 & [NeIII] & 386.9 \\
\hline 3 & 697.2 & 1.1 & 829 & 25 & [SII] & 672.5 & 12 & 615.1 & 0.8 & 2041 & 12 & $\mathrm{H} \delta$ & \\
\hline 4 & 553.4 & 3.1 & 6368 & 15 & MgII & 279.8 & 12 & 651.8 & 2.1 & 2018 & 19 & $\mathrm{H} \gamma$ & \\
\hline 5 & 402.2 & 2.3 & 1912 & 14 & MgII & 279.8 & 13 & 452.5 & 2.3 & 4206 & 27 & CIII] & 190.9 \\
\hline 5 & 623.8 & 3.8 & 2561 & 17 & $\mathrm{H} \gamma$ & & 13 & 666.8 & 1.0 & 1147 & 20 & MgII & 279.8 \\
\hline 5 & 698.9 & 8.0 & 2728 & 12 & $\mathrm{H} \beta$ & & 14 & 445.5 & 3.0 & 2641 & 25 & CIII] & 190.9 \\
\hline 5 & 712.2 & 1.5 & 771 & 12 & [OIII] & 495.9 & 14 & 653.3 & 0.9 & 1586 & 30 & MgII & 279.8 \\
\hline 5 & 718.9 & 2.4 & 431 & 12 & [OıII] & 500.7 & 16 & 502.5 & 2.0 & 1774 & 19 & MgII & 279.8 \\
\hline 6 & 397.5 & 1.0 & 871 & 12 & $\mathrm{Ly} \beta$ & & 17 & 422.7 & 1.6 & ..... & 13 & [OII] & 372.7 \\
\hline 6 & 468.2 & 10.5 & $2268^{*}$ & 16 & $\operatorname{Ly} \alpha$ & & 17 & 438.5 & 1.1 & 1344 & 14 & [NeIII] & 386.9 \\
\hline 6 & 539.2 & 0.4 & 1179 & 22 & SirV & 140.0 & 17 & 461.5 & 1.5 & 3277 & 16 & $\mathrm{H} \delta$ & \\
\hline 6 & 595.7 & 2.3 & 974 & 19 & Civ & 154.9 & 17 & 493.2 & 2.1 & 2878 & 14 & $\mathrm{H} \gamma$ & \\
\hline 7 & 449.2 & 4.7 & $4028^{*}$ & 35 & MgII & 279.8 & 17 & 551.3 & 3.6 & 3485 & 19 & $\mathrm{H} \beta$ & \\
\hline 7 & 598.0 & 0.1 & 662 & 21 & [OII $]$ & 372.7 & 17 & 562.1 & 2.6 & ..... & 18 & [ÖII] & 495.9 \\
\hline 7 & 697.8 & 1.1 & 2020 & 16 & $\mathrm{H} \gamma$ & & 17 & 567.7 & 5.8 & $\ldots .$. & 17 & [Оіні] & 500.7 \\
\hline 8 & 485.0 & 0.7 & 2619 & 19 & $\mathrm{H} \delta$ & & 17 & 714.6 & 0.7 & 667 & 13 & {$[\mathrm{OI}]$} & 630.0 \\
\hline 8 & 514.1 & 1.3 & 2521 & 17 & $\mathrm{H} \gamma$ & & 18 & 465.2 & 0.3 & ..... & 15 & $\mathrm{H} \gamma$ & \\
\hline 8 & 574.8 & 3.0 & 2347 & 18 & $\mathrm{H} \beta$ & & 18 & 520.8 & 1.3 & ..... & 28 & $\mathrm{H} \beta$ & \\
\hline 8 & 591.9 & 0.9 & 1137 & 22 & [OIII] & 500.7 & 18 & 531.4 & 0.8 & $\ldots .$. & 29 & [OIII] & 495.9 \\
\hline 9 & 397.7 & 0.6 & $\ldots .$. & 19 & [NeIII] & 386.9 & 18 & 536.5 & 2.3 & $\ldots .$. & 31 & [Öні] & 500.7 \\
\hline 9 & 421.9 & 0.3 & $\ldots .$. & 19 & $\mathrm{H} \delta$ & & 18 & 703.2 & 9.7 & 697 & 27 & $\mathrm{H} \alpha$ & \\
\hline 9 & 446.3 & 1.6 & $\ldots$. & 22 & $\mathrm{H} \gamma$ & & 18 & 720.2 & 1.9 & 852 & 33 & {$[\mathrm{SII}]$} & 672.5 \\
\hline
\end{tabular}

* Gaussian fit inappropriate/impossible for this line.

and by $36 \%$ in the annulus between $2^{\circ}$ and $4^{\circ}$ (see Fig. 2).

No less than 50 QSOs/AGNs with $B$ or $V \lesssim 18$ are now available behind Coma within a radius of $\approx 4^{\circ}$ of its centre, and they constitute the largest sample of QSOs/AGNs available behind a cluster, with a more uniform coverage from the inner Abell radius to the outskirts related to the large-scale filaments. Figure 2 gives their angular distribution and the positions of galaxies with known redshifts from a variety of surveys (Karachentsev \& Kopylov 1990; Huchra et al. 1995; Biviano et al. 1995; Doi et al. 1995; Donas et al. 1995; Colless \& Dunn 1996). Note that there are far many more galaxies known in this field (Godwin \& Peach 1977; Slezak et al. 1988), whose redshifts are likely to be measured in the near future by the Sloan Digital Sky Survey. Moreover, there are 15 QSO/AGN pairs with separations smaller than $30^{\prime}$. The sample thus provides ideal targets for observation with HST and for optical monitoring with small/medium-size telescopes to shed some light on the nature of the Lyman- $\alpha$ clouds at low redshift, to study the spatial distribution of the warm gas in and around the Coma cluster of galaxies, and its baryonic dark matter content.

Acknowledgements. We are grateful to A. Biviano, C. Lobo, F. Durret and M. Doi for providing galaxy and star positions in advance of publication, to M.-P. Véron-Cetty for clarifying the status of some rejected quasars, and to the CAI/MAMA team for their kind assistance. This research has made use of the SIMBAD database operated at CDS (Observatoire de Strasbourg, France) and of the NASA/IPAC Extragalactic Database (NED) operated by JPL (California Institute of Technology, U.S.A.). The OMHR survey is supported by the Programme National de Cosmologie (INSU/CNRS, France). The Hamburg Quasar Survey was supported by the Deutsche Forschungsgemeinschaft (DFG) under grants Re 353/11 and 22. The Hamburg/RASS identification project has been funded 


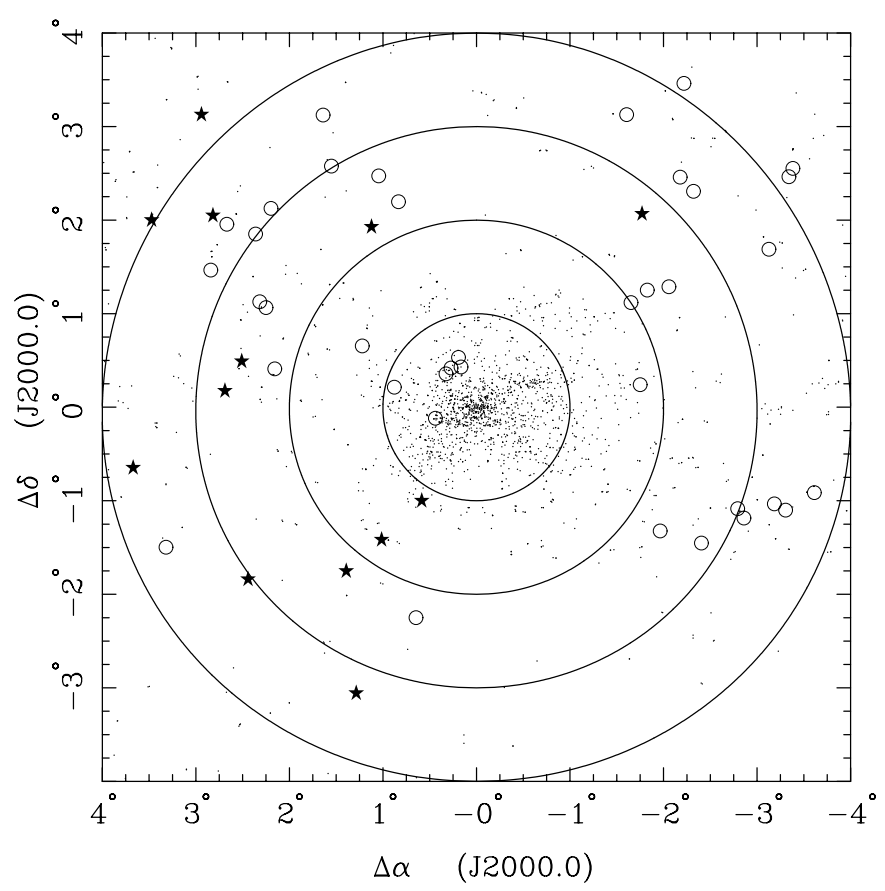

Fig. 2. Projected distribution of the new AGNs brighter than $B \approx 18$ behind Coma (filled stars) together with the AGNs listed in the compilation by Véron-Cetty \& Véron (1998) brighter than $V \approx 18$ in the same redshift range (empty circles). The dots indicate galaxies with measured redshifts within that solid angle (see text)

by the DFG under Re $353 / 22$ and by the BMBF under DARA 50 0R 96016.

\section{References}

Bade N., Engels D., Fink H., et al., 1992, A\&A 254, L21

Bade N., Engels D., Voges W., et al., 1998, A\&AS 127, 145

Baldwin J.A., Phillips M.M., Terlevich R., 1981, PASP 93, 5

Berger J., Cordoni J.P., Fringant A.M., et al., 1991, A\&AS 87, 389

Biviano A., 1998, in Untangling Coma Berenices: A new vision of an old cluster, Mazure A., Casoli F., Durret F., Gerbal D. (eds.). World Scientific Co., p. 1

Biviano A., Durret F., Gerbal D., et al., 1995, A\&AS 111, 265

Boller T., Brandt W.N., Fink H., 1996, A\&A 305, 53

Cen R., Miralda-Escudé J., Ostriker J.P., Rauch M., 1994, ApJ 437, L9

Colless M., Dunn A.M., 1996, ApJ 458, 435

D'Odorico V., Cristiani S., D'Odorico S., et al., 1998, A\&A 339,678

Dixon W.V.D., Hurwitz M., Ferguson H.C., 1996, ApJ 469, $\mathrm{L} 77$

Doi M., Fukugita M., Okamura S., Tarusawa K., 1995, ApJS 97, 77

Donas J., Milliard B., Laget M., 1995, A\&A 303, 661

Ensslin T.A., Biermann P.L., 1998, A\&A 330, 90

Fabian A.C., 1996, Sci 271, 1244

Godwin J.G., Peach J.V., 1977, MNRAS 181, 323
Hagen H.-J., Groote D., Engels D., Reimers D., 1995, A\&AS 111,195

Hagen H.-J., Engels D., Reimers D., 1999, A\&AS 134, 483

Hasinger G., Burg R., Giacconi R., et al., 1993, A\&A 275, 1

Hasinger G., Burg R., Giacconi R., et al., 1994, A\&A 291, 348

He X.-T., Cannon R.D., Peacock J.A., Smith M.G., Oke J.B., 1984, MNRAS 211, 443

Hernquist L., Katz N., Weinberg D.H., Miralda-Escudé J., 1996, ApJ 457, L51

Horne K., 1986, PASP 98, 609

Horowitz I.K., Moody J.W., Hintz E., 1994, AAS 185, 1902

Huchra J.P., Geller M.J., Corwin H.G.Jr., 1995, ApJS 99, 391

Impey C.D., He X.-T., 1986, MNRAS 221, 897

Karachentsev I.D., Kopylov A.I., 1990, MNRAS 243, 390

Kim K.-T., 1994, A\&AS 105, 403

Kim K.-T., Kronberg P.P., Dewdney P.E., Landecker T.L., 1994, A\&AS 105, 385

Knezek P.M., Bregman J.N., 1998, AJ 115, 1737

Koski A.T., 1978, ApJ 223, 56

Ledoux C., Valls-Gabaud D., Reboul H., et al., 1998, in Untangling Coma Berenices: A new vision of an old cluster, Mazure A., Casoli F., Durret F., Gerbal D. (eds.). World Scientific Co., p. 209

Lemaitre G., Kohler D., Lacroix D., Meunier J.P., Vin A., 1990, A\&A 228, 546

Lieu R., Mittaz J.P.D., Bowyer S., et al., 1996a, Sci 274, 1335

Lieu R., Mittaz J.P.D., Bowyer S., et al., 1996b, ApJ 458, L5

Miralda-Escudé J., Cen R., Ostriker J.P., Rauch M., 1996, ApJ 471,582

Moreau O., Reboul H., 1995, A\&AS 111, 169

Netzer H., 1990, in Active galactic Nuclei, Saas-Fee Advanced Course 20, p. 57

Oke J.B., 1990, AJ 99, 1621

Petitjean P., Mücket J.P., Kates R.E., 1995, A\&A 295, L9

Petitjean P., Surdej J., Smette A., et al., 1998, A\&A 334, L45

Sarazin C.L., Lieu R., 1998, ApJ 494, L177

Shanks T., Georgantopoulos I., Stewart G.C., et al., 1991, Nat 353,315

Shuder J.M., Osterbrock D.E., 1981, ApJ 250, 55

Slezak E., Mars G., Bijaoui A., Balkowski C., Fontanelli P., 1988, A\&AS 74, 83

Smette A., Robertson J.G., Shaver P.A., et al., 1995, A\&AS 113,199

Tadros H., Warren S., Hewett P., 1998, New Astron. Rev. 42, 115

Usher P.D., 1981, ApJS 46, 117

Veilleux S., Osterbrock D.E., 1987, ApJS 63, 295

Véron-Cetty M.P., Véron P., 1998, ESO Sci. Rep. 18, 1

Voges W., Aschenbach B., Boller T., et al., 1997, IAU Symp. 179,433

Walker M.A., Ireland P.M., 1995, MNRAS 275, L41

Weedman D.W., 1985, ApJS 57, 523

Wu X.-P., Xue Y.-J., 1998, Search for MACHOs in clusters of galaxies, Technical Report, Beijing Astronomical Observatory

Zickgraf F.-J., Thiering I., Krautter J., et al., 1997, A\&AS 123, 103 